\title{
Laparoscopic management of ureteral lesions in gynecology
}

\author{
Carlo De Cicco, M.D., ${ }^{\mathrm{a}, \mathrm{c}}$ Ron Schonman, M.D., ${ }^{\mathrm{a}}$ Marleen Craessaerts, ${ }^{\mathrm{a}}$ Ben Van Cleynenbreugel, M.D., ${ }^{\mathrm{b}}$ \\ Anastasia Ussia, M.D., ${ }^{\mathrm{d}}$ and Philippe Robert Koninckx, M.D., Ph.D. ${ }^{\mathrm{a}, \mathrm{c}}$ \\ ${ }^{a}$ Department of Obstetrics and Gynecology and ${ }^{b}$ Department of Urology, University Hospital Gasthuisberg, Katholieke \\ Universiteit Leuven, Leuven, Belgium; ${ }^{\mathrm{c}}$ Department of Obstetrics and Gynecology, University Hospital A. Gemelli, \\ Università Cattolica del Sacro Cuore, Rome, Italy; and ${ }^{\mathrm{d}}$ Department of Obstetrics and Gynecology, Villa Giose, Crotone, Italy
}

Objective: To investigate the outcome of laparoscopic repair of ureteral injury in laparoscopic gynecologic surgery.

Design: Prospective trial.

Setting: University hospital.

Patient(s): Forty patients with a ureteral lesion in laparoscopic surgery between 1991 and 2007.

Intervention(s): Laparoscopic ureteral repair, laparoscopic-assisted or blind stent insertion.

Main outcome measure(s): Treatment outcome of ureteral lesion analyzed by type of injury, time of diagnosis, and management.

Result(s): In 4,350 consecutive laparoscopic gynecologic interventions, 42 lesions occurred, 5 during hysterectomy, 1 during adnexectomy, and 36 during deep endometriosis surgery. In the latter group $(n=1,427)$, the incidence was $1.5 \%$ and $21 \%$ in women without and with hydronephrosis, respectively. In eight women in whom a stent was inserted after surgery without laparoscopic guidance, five were uneventful and three needed a second intervention. In all 34 women in whom a laparoscopic repair over a stent was performed, the outcome was uneventful, whether diagnosed and treated during surgery $(n=25)$ or after surgery $(n=9)$.

Conclusion(s): Laparoscopic repair over a stent was uneventful for all lacerations, transections, and fistulas, whether performed during or after surgery, and was superior to blind stent insertion. In women with hydronephrosis and deep endometriosis, a preoperative stent insertion seems to be mandatory. (Fertil Steril ${ }^{\circledR} 2008$; $\mathbf{\square}: \mathbf{\square}$. (C)2008 by American Society for Reproductive Medicine.)

Key Words: Ureteral injury, ureteral repair, gynecologic surgery, endometriosis, hysterecomy, laparoscopy

Gynecologic surgery is associated with ureteral lesions, with an incidence ranging from 0.1 to $1.5 \%$ for benign procedures and up to $5 \%$ for oncologic procedures $(1-4)$. Whether laparoscopic procedures increase the risk of ureteral lesion is unclear, although a higher risk of ureteral and other complications can be expected during the learning curve (5).

The management of ureteral lesions might be at a turning point by the introduction of laparoscopy. Traditionally, ureteral lesions are treated by laparotomy. Lacerations are treated with a double $\mathbf{J}$ stent with or without a suture. When this fails or after transections, ureteral reimplantation in the bladder $(1,6,7)$, with or without a Boari flap, is generally performed. Ureteral reanastomosis, although reported to have an overall success rate of $94 \%$, never gained widespread popularity (1). Over the last decade, laparoscopically aided treatment of ureteral injuries, such as the removal of a suture or stricture, the laparoscopically guided stent insertion and suturing for ureteral laceration, the laparoscopic reanastomo-

Received May 10, 2008; revised July 21, 2008; accepted August 7, 2008. C.D.C. has nothing to disclose. R.S. has nothing to disclose. M.C. has nothing to disclose. B.V.C. has nothing to disclose. A.U. has nothing to disclose. P.R.K. has nothing to disclose.

Reprint requests: Carlo De Cicco, Department of Obstetrics and Gynaecology, University Hospital Gasthuisberg, Herestraat 49 bus 611, B3000 Leuven, Belgium (FAX: +0039 06 233215178; E-mail: carlodecicco@gmail.com). sis over a stent, and, very recently, ureter reimplantations have become feasible $(2,8-11)$. The numbers, however, are still small and do not permit solid conclusions.

To contribute to this, we therefore reviewed treatment and outcome of all our ureteral iatrogenic lesions between 1991 and 2007.

\section{MATERIALS AND METHODS Database Review}

Our database is a self-developed Visual FoxPro relational database in which all interventions performed by P.R.K. since 1988 have been entered immediately after surgery and completed for postoperative complications. All records have been reviewed for eventual missing events and updated accordingly. The records of all patients with a ureteral lesion were reviewed in detail to ascertain the type of injury (occlusion, laceration, or section), the localization, the day of recognition, how diagnosis was made, the management, and the final outcome.

\section{Diagnosis of Ureteral Lesions}

Ureteral lesions were diagnosed either during surgery by direct visualization or after surgery by intravenous pyelography (IVP) or computerized tomographic (CT) scan, or during a repeat laparoscopy which was performed systematically 
whenever the condition of the patient did not improve progressively after surgery.

\section{Treatment of Ureteral Lesions}

All treatments of ureteral lesions were performed in collaboration with the department of urology, i.e., those with a specific interest in endoscopic surgery, initially Hans Goethuys (H.G.) and later B.V.C. When diagnosed intraoperatively and when judged feasible, primary repair was attempted by laparoscopy since 1991 . When diagnosis was made after surgery, treatment has varied over time according to the diagnosis and the presence of a laparoscopically trained urologist.

Intraoperatively a laceration was treated with double $\mathbf{J}$ stent insertions and a suture, i.e., in the early years polyglactin 910 3-0 (Vicryl; Ethicon, Somerville, NJ) and, after 1999, polyglecaprone 25 5-0 (Monocryl; Ethicon), using $3 \mathrm{~mm}$ instruments (Koh set, Storz, Tuttlingen, Germany). Transections were repaired by reanastomosis after oblique section of the ureter ends and insertion of a stent using four or five stitches of polyglecaprone 25 5-0 (Monocryl). When diagnosed after surgery, treatment was similar when both P.K. and H.G./B.V.C. were available; otherwise treatment remained traditional, i.e., a blind stent insertion, eventually associated with a nephrostomy.

\section{Statistics}

Significance was evaluated with chi-squared or Fisher exact test.

\section{RESULTS \\ Incidence}

A total of 42 lesions (in 40 patients) were retrieved. One woman was referred for ureteral transection after hysterec- tomy, and 41 occurred in a series of 4,350 consecutive gynecologic laparoscopic procedures, which is an overall incidence of $1 \%$. In 1,427 interventions for deep rectovaginal or sigmoidal endometriosis, we had 1 ligation and 35 lesions, 14 in 67 women $(21 \%)$ with and 21 in 1,360 women $(1.5 \%)$ without hydronephrosis $(P<.0001$, Fisher exact). In 430 hysterectomies, four lesions occurred and an ureteral coagulation occurred during adnexectomy. This series comprised 27 lacerations, 7 transections, 4 stricture/coagulations, 3 fistulas, and 1 ligation (Table 1).

In the 36 injuries which occurred during endometriosis surgery, 3 were unavoidable because of transmural endometriosis necessitating partial ureteral resection, 1 was a ligation during an extensive bladder suture (discovered at cystoscopy and deligated), 2 were strictures following devascularisation, and 30 were lacerations or transections.

Left ureteral lesions were more frequent in deep endometriosis surgery ( $P=.03$, Fisher exact), 22 being on the left side, 10 on the right side, and 2 bilateral. During hysterectomy the right ureter was injured in all five cases.

\section{Diagnosis}

Diagnosis was made during surgery in 25 out of 42 lesions, i.e., in 19 of 27 lacerations, 3 of 7 transection, 2 of 4 obstructions, and the single case of ligation. After surgery, diagnosis was made in four woman within 2 days, in six women between days 3 and 5, in two between days 6 and 10, in three between days 10 and 20, and after 28 and 29 days in the remaining two (Fig. 1). The diagnosis was made by urine leakage from the drain in six or from the vagina in two women, by IVP or CT scan in five. and by repeat laparoscopy in four. Early repeat laparoscopy was a standard procedure

\section{TABLE 1}

\section{Management and outcome of ureteral lesions.}

\begin{tabular}{|c|c|c|c|c|}
\hline \multirow[b]{2}{*}{ Type of lesion } & \multirow[b]{2}{*}{ Treatment } & \multicolumn{2}{|c|}{$\begin{array}{l}\text { Laparoscopic repair or } \\
\text { laparoscopic-aided stent insertion }\end{array}$} & \multirow{2}{*}{$\frac{\text { Blind stenting }}{\text { After surgery }}$} \\
\hline & & During surgery & After surgery & \\
\hline Obstruction & Stent & $2(2)$ & - & $2(2)$ \\
\hline Ligation & Deligation & $1(1)$ & - & - \\
\hline Pinpoint laceration & Stent & $2(2)$ & - & - \\
\hline \multirow[t]{2}{*}{ Laceration } & Stent/nephrostomy & - & - & $5(3)^{a}$ \\
\hline & Stent + suture & $17(17)$ & $3(3)$ & - \\
\hline Transection & Stent + suture & $3(3)$ & $4(4)$ & - \\
\hline \multirow{2}{*}{ Fistula } & Stent/nephrostomy & - & - & $1(0)^{b}$ \\
\hline & Stent + suture & - & $2(2)$ & - \\
\hline Total & & $25(25)$ & $9(9)$ & $8(5)$ \\
\hline \multicolumn{5}{|c|}{$\begin{array}{l}\text { Note: Values in parentheses are number with uneventful outcome. } \\
\text { a Two patients required laparotomic reimplantation: one for persistent leakage and the second for ureterovaginal fistula } \\
\text { formation. } \\
\text { b One patient required reimplantation after fistula's persistence. }\end{array}$} \\
\hline \multicolumn{5}{|c|}{ De Cicco. Laparoscopic ureteral repair is feasible. Fertil Steril 2008.} \\
\hline
\end{tabular}




\section{FIGURE 1}

Ureteral lesions diagnosed after surgery. The type of lesion and the delay in diagnosis are indicated.

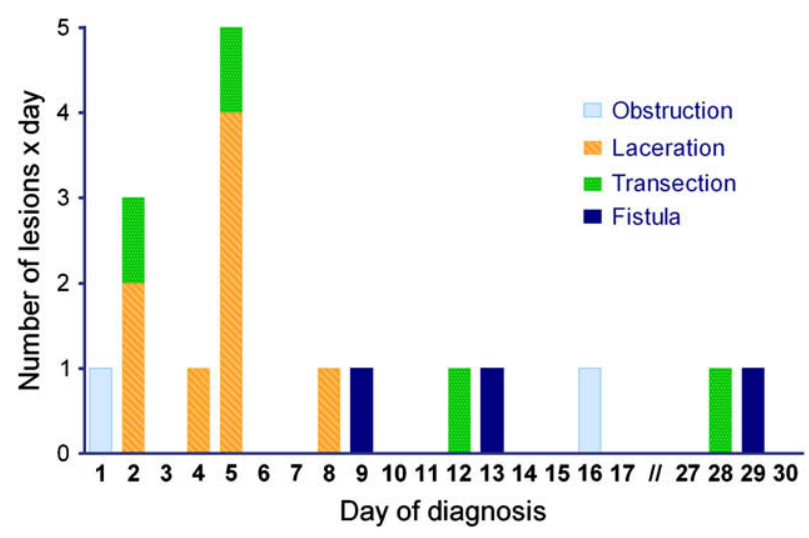

De Cicco. Laparoscopic ureteral repair is feasible. Fertil Steril 2008.

performed whenever judged to be clinically indicated. When the clinical symptoms were insufficient to warrant a repeat laparoscopy but too important to ignore, an IVP or CT scan was performed.

\section{Treatment and Outcome}

All 25 lesions diagnosed during surgery were treated by laparoscopic repair $(n=21)$ or by laparoscopic-aided stent insertion $(n=4)$, and outcome was uneventful in all; 17 lacerations were stented and sutured, three transections were treated by reanastomosis, one ligation was deligated and stented, and four prophylactic stents were placed (two for pinpoint laceration and two for ureteral devascularization).

Of the 17 lesions diagnosed after surgery, nine were treated by laparoscopic repair and eight by blind stent insertion. All laparoscopic repairs had an uneventful outcome, even in women in whom the diagnosis had been delayed for several days; three lacerations were managed by stenting and suturing, four transections underwent reanastomosis, and two fistulas were dissected and sutured over a stent. Blind stent insertion was performed when the laparoscopic team was not available or, in the beginning, when the laparoscopic expertise was lower, i.e., in five lacerations, two obstructions, and one fistula. In five of these, stent insertion was sufficient, but three required further surgery. In one woman, with a laceration treated by blind stenting, leakage persisted and 6 weeks later a reimplantation was performed. One patient, in whom a laceration was initially treated by blind stenting for 11 days, developed an ureterovaginal fistula; a subsequent attempt of laparoscopic repair failed, requiring ureteral reimplantation. Another patient with a fistula required reimplantation 12 weeks after blind stent insertion, owing to persistent leakage.

The outcome of treatment, whether performed during surgery or after a delayed diagnosis, did not differ significatively.
Comparing primary laparoscopic management with blind stent insertion, the former was superior. All lesions treated during surgery by laparoscopy $(n=21)$ or by a laparoscopically aided stent insertion $(n=4)$ had an uneventful outcome, as well as the lesions diagnosed after surgery $(n=9)$ when treated by laparoscopy. Out of the eight women treated after surgery by blind stenting, two obstructions had an uneventful outcome, whereas three of the six women with leakage or fistula required a subsequent reimplantation. Laparoscopic management was superior to blind stent insertion in the laceration group ( $P=.03$, Fisher exact) and in the postoperative leakage group ( $P=.04$, Fisher exact). Furthermore, outcome of laparoscopic repair and laparoscopic aided stent insertion were, together, far superior to blind stenting ( $P=.005$, Fisher exact).

\section{DISCUSSION}

An overall 1.5\% of ureteral lesions in deep endometriosis surgery without hydronephrosis is similar to the overall reported incidences of ureteral lesions between 0.5 and $4 \%(1-4,12)$. As can be expected, but to the best of our knowledge not yet reported, the incidence increases when hydroureteronephrosis is present. The observation that more lesions occur at the left side is not surprising, because severe endometriosis has been reported to occur more frequently on the left side (13). A full analysis of the relationship between the size of the nodules, the difficulty of surgery, and the expertise of the surgeon is beyond the scope of the present report.

The data confirm that outcome of lacerations treated by a suture over a stent is superior to stenting only, as was concluded in our recent review (1). Taking together all data from the present series and from the earlier review, stenting with suturing was uneventful in all 32 women, whereas stenting only required a further intervention in 6 out of 14 women $(P=.0003)$. For pinpoint lesions stenting will probably be sufficient. Because we had the impression, from laparoscopic-aided stenting, that blind stenting sometimes carries the risk of enlarging small lacerations, we suggest the use of laparoscopic-aided stenting in all cases of postoperative leakage.

The present results also confirm the successful outcome of laparoscopic reanastomosis to treat ureteral transections. The data from the earlier review together with the present series show that laparoscopic reanastomosis was successful in all 29 women.

The only failure of a laparoscopic suture over a stent was in a case of ureterovaginal fistula in which a secondary repair was attempted, 17 days after surgery, in a woman who had a stent already for 11 days. Stitching was described as "difficult" because of edematous tissue. Considering the easy suture in a woman 4 days after stenting, we suggest that the delay between stent insertion and laparoscopic repair by suturing should not be too long.

These are, to the best of our knowledge, the first two ureterovaginal fistulas successfully treated by laparoscopic 
dissection and repair. The numbers are obviously too small to discuss the technique, which consisted of a five-stitch circular, almost watertight, suture of the ureter, a two-layer watertight running suture of the vagina, and interposition of the omentum.

Our data confirm that obstructive lesions can be managed by stenting only. The present data, moreover, strongly suggest, as was tentatively concluded in a review (1), that the outcome of laparoscopic treatment of lacerations or of transections do not differ whether diagnosed during or after surgery. The uneventful outcome of a reanastomosis 28 days after surgery was surprising, as was the fact that surgery was technically easy. Although we do not know yet how long ureteral repair can safely be postponed, we may conclude that a ureteral repair can be safely postponed for several days if necessary, eventually permitting referral if necessary. In any case, the widely held belief that delayed diagnosis impairs outcome seems not to be substantiated.

These results strongly suggest that laparoscopic treatment has become an option for the repair of ureteral lesions, not only when diagnosed during surgery, but also when diagnosed after surgery. Results are similar to the traditional reimplantation of the ureter with or without psoas hitch, which also is close to $100 \%$ effective. Considering, however, the minimal invasiveness of a laparoscopic treatment, this definitively is becoming the preferred technique for ureteral lacerations and transections (8). There are no data supporting what should be done when the first-line laparoscopic treatment fails, because a second laparoscopic intervention was never attempted.

Whether the liberal use of preoperative stenting should be advocated in deep endometriosis surgery is unclear. Some surgeons almost routinely use preoperative stenting, whereas others only use stenting when a hydronephrosis is diagnosed preoperatively, when during surgery an extensive dissection of the ureter over more than $6 \mathrm{~cm}$ is performed, or when some coagulation damage to the ureter is suspected. Those in favor of liberal stenting suggest that transection of the ureter will not go unnoticed during surgery, and that some of our postoperatively diagnosed leaks would not have occurred. Indeed, Figure 1 and the clinical history strongly suggest that at least in some of these women, leakage only started after 3-6 days, thus introducing the knowledge of late leakage as known for bowel perforations (14). Moreover, small lacerations can be treated by stenting only, and thus will go unnoticed when stented prophylactically. Stenting, however, is not without side effects. First, some women suffer after surgery from pain caused by the stent, and the risk of infection requires the prophylactic use of urinary antiseptica. Second, although data to substantiate this are hard to find, many believe that repetitive use of a stent transforms the ureter into a rigid organ with a thickened wall without much contractions. How important this is and whether these women are prone to retrograde infection and pyelonephritis is not clear. We suggest that preoperative stenting is indicated in women with deep endometriosis and hydronephrosis given a $21 \%$ risk of ure- teral lesions. In women without hydronephrosis, however, with a risk of unnoticed lesions of $<0.5 \%$, even for severe surgery, liberal use of stenting is not indicated. In any case, the subjective feeling of safety when a stent has been inserted or the idea that pelvic dissection is facilitated seems to be controversial.

In conclusion, unintended ureteral injuries occur in some $1.5 \%$ during deep endometriosis surgery without hydronephrosis and in $21 \%$ when hydronephrosis is present. The use of a preoperative ureteral stent seems to be indicated when hydronephrosis is present, otherwise we favor the use of stents upon indication only. Most importantly, we confirm that laparoscopic management of ureteral lesions could become the preferred first line of treatment whether diagnosed during or after surgery. Whether this also applies to the laparoscopic management of ureterovaginal fistula is unknown, but at least the feasibility is established.

Acknowledgments: The authors thank Roberta Corona, Jasper Verguts, and George Betsas, Leuven, Belgium, and Fiorenzo De Cicco, Rome, Italy, for reviewing this manuscript. Diane Wolput, Leuven, Belgium, is also thanked for helping in data collection. The Catholic University of Leuven and the Catholic University of Rome are acknowledged for making this possible through the exchange program between both universities.

\section{REFERENCES}

1. De Cicco C, Ret Davalos ML, Van Cleynenbreugel B, Verguts J, Koninckx PR. Iatrogenic ureteral lesions and repair: a review for gynecologists. J Minim Invasive Gynecol 2007;14:428-35.

2. Ou CS, Huang IA, Rowbotham R. Laparoscopic ureteroureteral anastomosis for repair of ureteral injury involving stricture. Int Urogynecol $\mathrm{J}$ Pelvic Floor Dysfunct 2005;16:155-7.

3. Ostrzenski A, Radolinski B, Ostrzenska KM. A review of laparoscopic ureteral injury in pelvic surgery. Obstet Gynecol Surv 2003;58:794-9.

4. Chan JK, Morrow J, Manetta A. Prevention of ureteral injuries in gynecologic surgery. Am J Obstet Gynecol 2003;188:1273-7.

5. Assimos DG, Patterson LC, Taylor CL. Changing incidence and etiology of iatrogenic ureteral injuries. J Urol 1994;152:2240-6.

6. Benoit L, Spie R, Favoulet P, Cheynel N, Kretz B, Gouy S, et al. [Management of ureteral injuries]. Ann Chir 2005;130:451-7. French.

7. Png JC, Chapple CR. Principles of ureteric reconstruction. Curr Opin Urol 2000;10:207-12.

8. Rassweiler JJ, Gozen AS, Erdogru T, Sugiono M, Teber D. Ureteral reimplantation for management of ureteral strictures: a retrospective comparison of laparoscopic and open techniques. Eur Urol 2007;51:512-22.

9. Rafique M, Arif MH. Management of iatrogenic ureteric injuries associated with gynecological surgery. Int Urol Nephrol 2002;34:31-5.

10. Sakellariou P, Protopapas AG, Voulgaris Z, Kyritsis N, Rodolakis A, Vlachos G, et al. Management of ureteric injuries during gynecological operations: 10 years experience. Eur J Obstet Gynecol Reprod Biol 2002;101:179-84.

11. Liapis A, Bakas P, Giannopoulos V, Creatsas G. Ureteral injuries during gynecological surgery. Int Urogynecol J Pelvic Floor Dysfunct 2001;12: 391-3.

12. Slack A, Child T, Lindsey I, Kennedy S, Cunningham C, Mortensen N, et al. Urological and colorectal complications following surgery for rectovaginal endometriosis. BJOG 2007;114:1278-82.

13. Al Fozan H, Tulandi T. Left lateral predisposition of endometriosis and endometrioma. Obstet Gynecol 2003;101:164-6.

14. Koninckx PR, Timmermans B, Meuleman C, Penninckx F. Complications of $\mathrm{CO}_{2}$-laser endoscopic excision of deep endometriosis. Hum Reprod 1996;11:2263-8. 